\title{
A Determination of the $\mathrm{C} / \mathrm{M}^{+}$Ratio in the Galactic Plane
}

\author{
MARIA G. NIKOLASHVILI \\ Abastumani Astrophysical Observatory \\ 383762 Abastumani, Republic of Georgia
}

Two deep low-dispersion objective-prism spectral surveys, in the yellowred and near-infrared spectral regions, have been carried out at Abastumani Astrophysical Observatory for the identification of late-type stars, especially carbon stars, with the $70-\mathrm{cm}$ meniscus telescope equipped with a $2^{\circ}$ prism (1250 $\AA \mathrm{mm}^{-1}$ at $H_{\gamma}$ and $7000 \AA \mathrm{mm}^{-1}$ at the A-band). As a result of both surveys about 2200 carbon stars have been identified, among them more than 1400 new ones. On the basis of these spectral surveys the $\mathrm{C} / \mathrm{M}^{+}$ratios have been determined in 100 survey fields located at latitudes $b=0^{\circ}, \pm 3.6$. The numbers of $\mathrm{M}$ stars were counted in $2^{\circ} \times 2^{\circ}$ squares. The results are given in

\begin{tabular}{crccccrccc}
\hline Region & New & All & N/S & C/M5 $^{+}$ & Region & New & All & N/S & $\mathrm{C}^{-M^{+}}$ \\
\hline $30-50$ & 85 & 111 & 0.56 & 0.02 & $115-130$ & 115 & 181 & 1.12 & 0.09 \\
$50-70$ & 145 & 217 & 1.09 & 0.04 & $130-145$ & 79 & 122 & 0.81 & 0.14 \\
$70-90$ & 156 & 264 & 1.32 & 0.06 & $145-165$ & 83 & 172 & 0.86 & 0.25 \\
$90-115$ & 146 & 278 & 1.11 & 0.09 & $195-210$ & 53 & 158 & 1.005 & 0.30 \\
\hline
\end{tabular}

the table. As is seen, the $\mathrm{C} / \mathrm{M}^{+}$ratios vary from 0.02 to 0.30 as the longitude varies from $30^{\circ}$ to $210^{\circ}$. It is well known that in the galaxies of the Local Group this ratio is correlated with the metal abundance of those systems. It might be noted that this ratio is equal to 0.7 and 4.4 for the LMC and SMC, respectively (Richer, 1989, in IAU Coll. 106, Evolution of Peculiar Red Giant Stars, p. 35). 